\title{
21
}

\section{An Improved Water Distribution System Chlorine Decay Model Using EPANET}

MSX

\author{
Walter Grayman, Sudhir Kshirsagar, Melixa Rivera-Sustache and \\ Mark Ginsberg
}

One of the most common uses of water distribution system water quality modeling is to simulate the chlorine residual in the network. Most standard software packages such as EPANET model the decay of chlorine using a bulk decay rate and a wall decay coefficient. In EPANET, the bulk decay rate is either defined as a single constant for the entire distribution system or is assigned different fixed values for specific pipes. If there are multiple sources within a distribution system that produce finished water with different chlorine decay characteristics, it is not possible within EPANET to accurately represent the decay in areas of the system where the water from multiple sources is blended.

The multiple species extension (MSX) to EPANET allows the user to create a simple text file (MSX file) that more accurately represents the bulk decay when there are multiple sources with different decay rates. This chapter describes how an MSX file was developed that first calculated the percentage of water coming from each source to each pipe over time, and secondly used the percentages to calculate a bulk decay rate for each pipe for each time step as a weighted combination of the bulk decay rate for each of the multiple sources.

This MSX representation was tested and demonstrated using the standard EPANET NET3 distribution system example, and is currently being applied to a water distribution system at an army base. Similar MSX representations can be written to simulate other parameters such as disinfection byproducts.

Grayman, W., S. Kshirsagar, M. Rivera-Sustache and M. Ginsberg. 2012. "An Improved Water Distribution System Chlorine Decay Model Using EPANET MSX." Journal of Water Management Modeling R245-21. doi: 10.14796/JWMM.R245-21.

C CHI 2012 www.chijournal.org ISSN: 2292-6062 (Formerly in On Modeling Urban Water Systems. ISBN: 978-0-9808853-7-8) 


\subsection{Introduction}

\subsubsection{Problem Definition}

Water quality modeling is an extension to hydraulic modeling that can be used to predict the movement and transformation of water quality parameters in a water distribution system. EPANET and most commercial network modeling software packages have a pre-defined set of water quality routines that can be used to model chlorine decay, disinfection byproduct formation and any conservative water quality parameters. Although these are a powerful set of water quality routines, they limit the user to the specific mechanisms that are supported by the software.

Chlorine decay is one of the most common applications of water quality modeling. EPANET and most commercial software packages model the decay of chlorine using a bulk decay rate and a wall decay coefficient (Rossman et al., 1994). Bulk decay accounts for the loss of chlorine corresponding to the water aging within the distribution system; it is typically considered to be independent of the type of conveyance or storage mechanism. Wall decay is the loss of chlorine due to the interaction of the flowing water with the pipe wall.

The rates of decay associated with the two mechanisms are controlled by a bulk decay rate coefficient and a wall decay coefficient. Typically, the bulk decay rate is calculated based on bottle tests for each source of water. For the case of a single water source, the decay rate is unilaterally assigned to the entire distribution system. If there are multiple sources, the decay rate is assigned to the areas served by each source. The wall decay coefficient is generally assigned individually to pipes based on their characteristics (e.g. pipe material, pipe age or pipe diameter) and may be further calibrated based on field tests.

One significant limitation of the standard bulk decay formulation in EPANET can occur if there are multiple sources. Specifically, if the bulk decay rate varies from one source to another and if there are mixing zones in the distribution system which receive water from different zones at different times, then there is no direct mechanism for modeling this variation in EPANET. The work described in this chapter utilizes a relatively new addition to EPANET called the multi species extension (MSX) and provides a formulation for assigning bulk decay rates dynamically to individual pipes based on the source of the water in the pipe at any given time.

\subsubsection{Multi Species Extension to EPANET}

MSX is an extension to the original EPANET that allows it to model any system of multiple, interacting chemical species (Shang et al., 2008). This 
capability is available as a standalone executable program as well as a toolkit library of functions that programmers can use to build custom applications. When applying MSX, an MSX input file is prepared by the user that defines the individual water quality species of interest and the reaction expressions that govern their dynamics. A partial example of an MSX input file is shown later in this chapter.

\subsubsection{Revised Chlorine Decay Model}

Using the MSX capability, a revised method for dynamically calculating and assigning chlorine bulk decay coefficients to individual pipes was developed. In this method, the percentage of water from each source reaching each pipe during each time step is calculated. The bulk decay coefficient is then calculated for each pipe and time step as a linear combination of bulk decay coefficients from each source, weighted by the fraction of water reaching each pipe from each source during a each time step. This is illustrated by equation 21.1 for the case of two sources.

$$
K b(i, t)=[K B 1 \cdot T R 1(i, t)]+[K B 2 \cdot \operatorname{TR} 2(i, t)]
$$

where:

$$
\begin{aligned}
K b(i, t)= & \text { bulk coefficient for pipe } i \text { at time } t \\
K B 1= & \text { bulk coefficient for water from source } 1, \\
K B 2= & \text { bulk coefficient for water from source } 2, \\
T R 1(i, t)= & \text { fraction of water in pipe } i \text { from source } 1 \text { at time } t, \\
T R 2(i, t)= & \text { fraction of water in pipe } i \text { from source } 2 \text { at time } t, \text { and } \\
& \operatorname{TR} 1(i, t)+\operatorname{TR} 2(i, t)=1
\end{aligned}
$$

The method can be generalized for any number of water sources.

\subsection{Case Study}

\subsubsection{Description of Case Study Distribution System}

The case study network used in this chapter is the NET3 example network that is distributed with the EPANET software and has been used widely in the literature to demonstrate EPANET applications. This network is a skeletonized representation of part of an actual unnamed water distribution system.

The network is composed of 92 junctions, 2 reservoirs (representing sources), 3 tanks, 117 pipes and 2 pumps. The average water use in the system is $15.8 \mathrm{MGD}(691.25 \mathrm{~L} / \mathrm{s})$ with 4.7 MGD $(205.63 \mathrm{~L} / \mathrm{s})$ used in the pressure zone represented by the model and 21.1 MGD $(485.63 \mathrm{~L} / \mathrm{s})$ exported to adjacent pressure zones. The total length of pipe in the system is $215712 \mathrm{ft}(65749 \mathrm{~m})$. 
The two sources of water, a river and a lake, have significantly different water quality characteristics with the better quality river water supplying about $82 \%$.

Figure 21.1 is a schematic representation of the water distribution system as represented in the model. The locations of the two sources and three tanks are shown. EPANET was used to trace the water from the two sources and the average percentages of water reaching different parts of the distribution system are showing by the shaded areas.

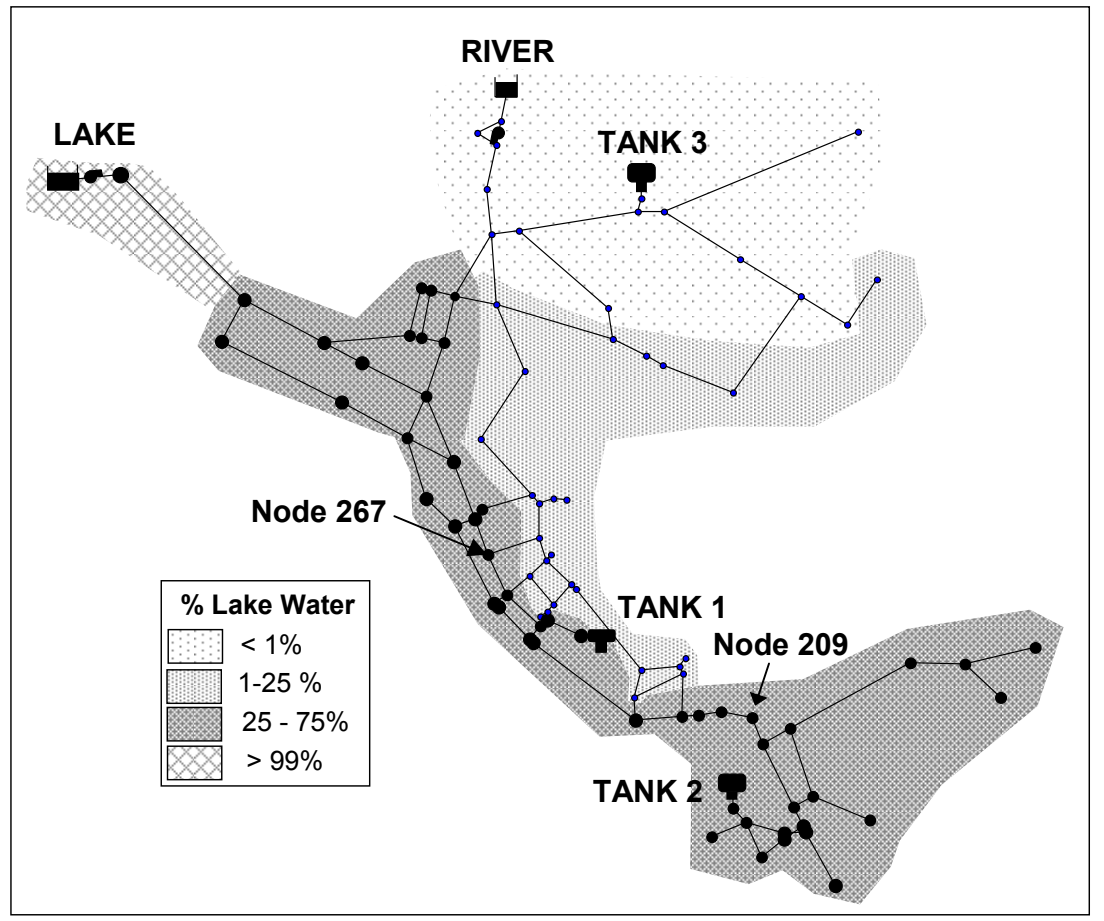

Figure 21.1 Case study distribution system showing average percentage of water emanating from lake source.

Because of the temporal variation in water use in the system over the course of the day and the different operational patterns for the two sources, the percentage of water from the lake source reaching different parts of the distribution system varies significantly by time of day. As an example, the temporal variation of the percentage of water from the lake reaching node 267 is shown in Figure 21.2. The location of node 267 is shown in Figure 21.1 above. 


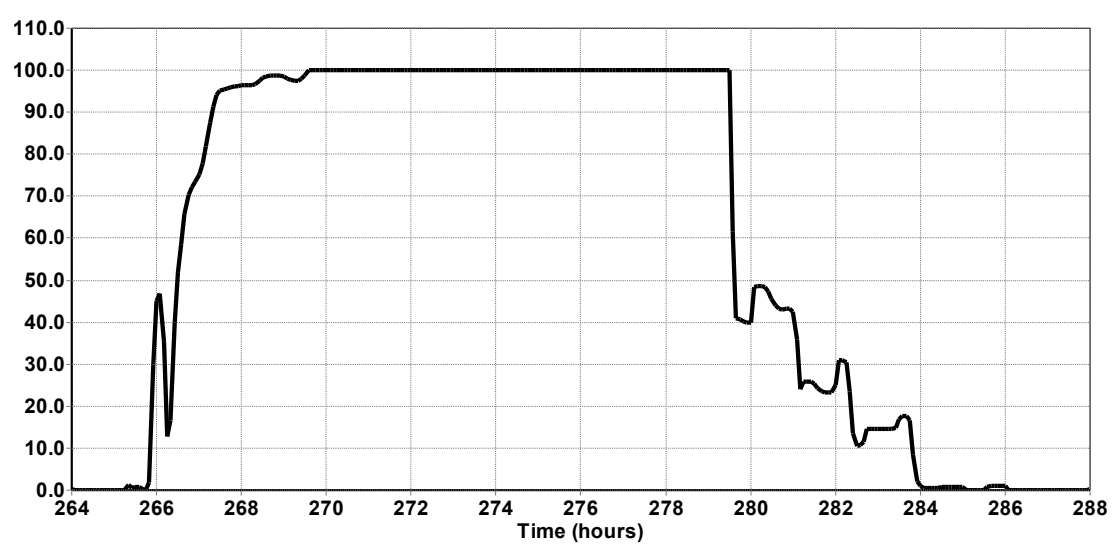

Figure 21.2 Percentage of lake water reaching node 267 over the course of an average day.

As will be illustrated in the following sections, the very significant temporal and spatial variations in the percentage of water from the two different sources introduces significant complexities in modeling the bulk chlorine decay processes in the distribution system.

\subsubsection{Application of Alternative Chlorine Decay Models}

Three alternative models were used to represent the bulk chlorine decay process in the case study distribution system:

global (constant) chlorine bulk decay coefficient;

zoned chlorine bulk decay; and

chlorine bulk decay coefficient dynamically calculated and assigned to each pipe based on the percentage of water emanating from each source.

The first two models used the capabilities available in the standard EPANET software while the third method used the capabilities available in EPANET MSX.

The bulk decay coefficients for the two sources of water used in this study were $-0.5 / \mathrm{d}$ for the river water and $-5.0 / \mathrm{d}$ for the lake water. This ratio of bulk chlorine decay coefficients between the two sources of $1: 10$ is representative of historical values for that system (Vasconcelos et al., 1996).

For the first model, a single flow-weighted global bulk chlorine decay value was calculated and applied for the entire system. The single global value used was $-1.2 / \mathrm{d}$. For the second model, the distribution system was divided into five zones based on the average percentage of water emanating from the two sources as shown in Figure 21.1 above. The assigned zonal values were: 
Zone $\mathrm{A}:<1 \%$ water from lake, $\mathrm{Kb}=-0.5 / \mathrm{d}$;

Zone B: $1 \%$ to $25 \%$ water from lake, $\mathrm{Kb}=-1.0 / \mathrm{d}$;

Zone $\mathrm{C}: 25 \%$ to $75 \%$ water from lake, $\mathrm{Kb}=-2.75 / \mathrm{d}$;

Zone D: $75 \%$ to $99 \%$ water from lake, no links in this zone; and

Zone $\mathrm{E}:>99 \%$ water from lake, $\mathrm{Kb}=-5.0 / \mathrm{d}$.

For the third model, EPANET MSX was used to model the revised bulk chlorine decay formulation described by equation 21.1. The multiple species used in the MSX modeling are the fraction of water coming from the two sources and the resulting calculated chlorine residual. The key parts of the MSX input file used to dynamically calculate the bulk chlorine decay based on the fraction of water coming from each source at each time step are shown in Figure 21.3.

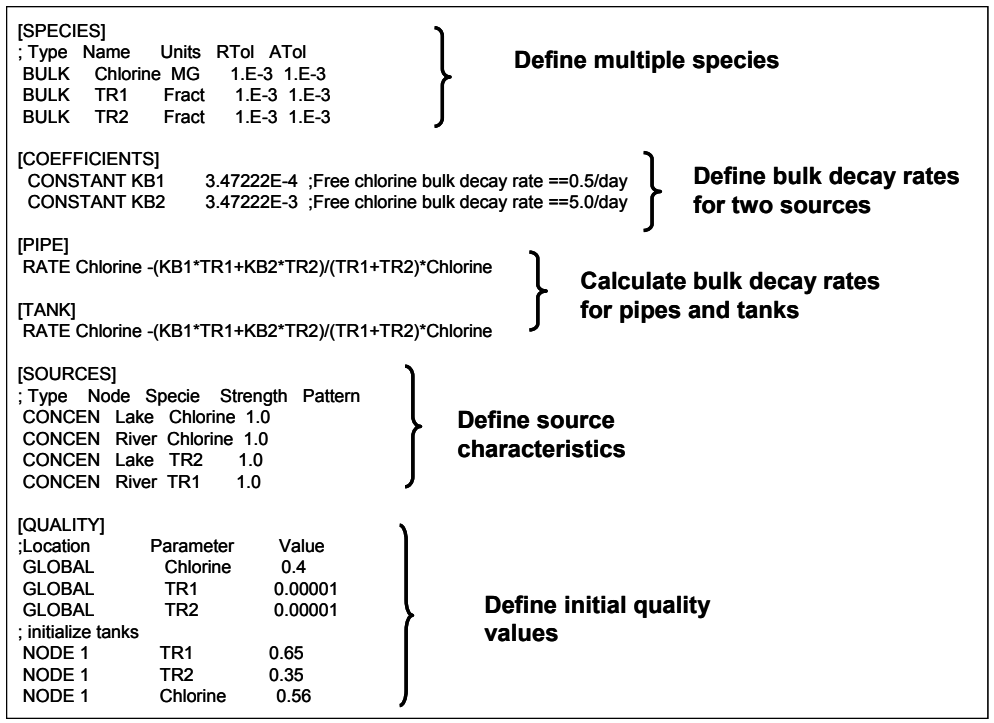

Figure 21.3 Example of partial MSX input file describing bulk chlorine decay model.

\subsubsection{Case Study Results}

The three alternative chlorine models were applied under the same hydraulic conditions and the resulting chlorine residual time series determined and analyzed. Figure 21.4 shows the modeled chlorine residual results over a $24 \mathrm{~h}$ period at the three tanks. Figure 21.5 presents similar results at nodes 267 and 209 (see Figure 21.1 for location of nodes and tanks). Table 21.1 presents the resulting calculated range of chlorine residuals at the tanks and at the two nodes. 

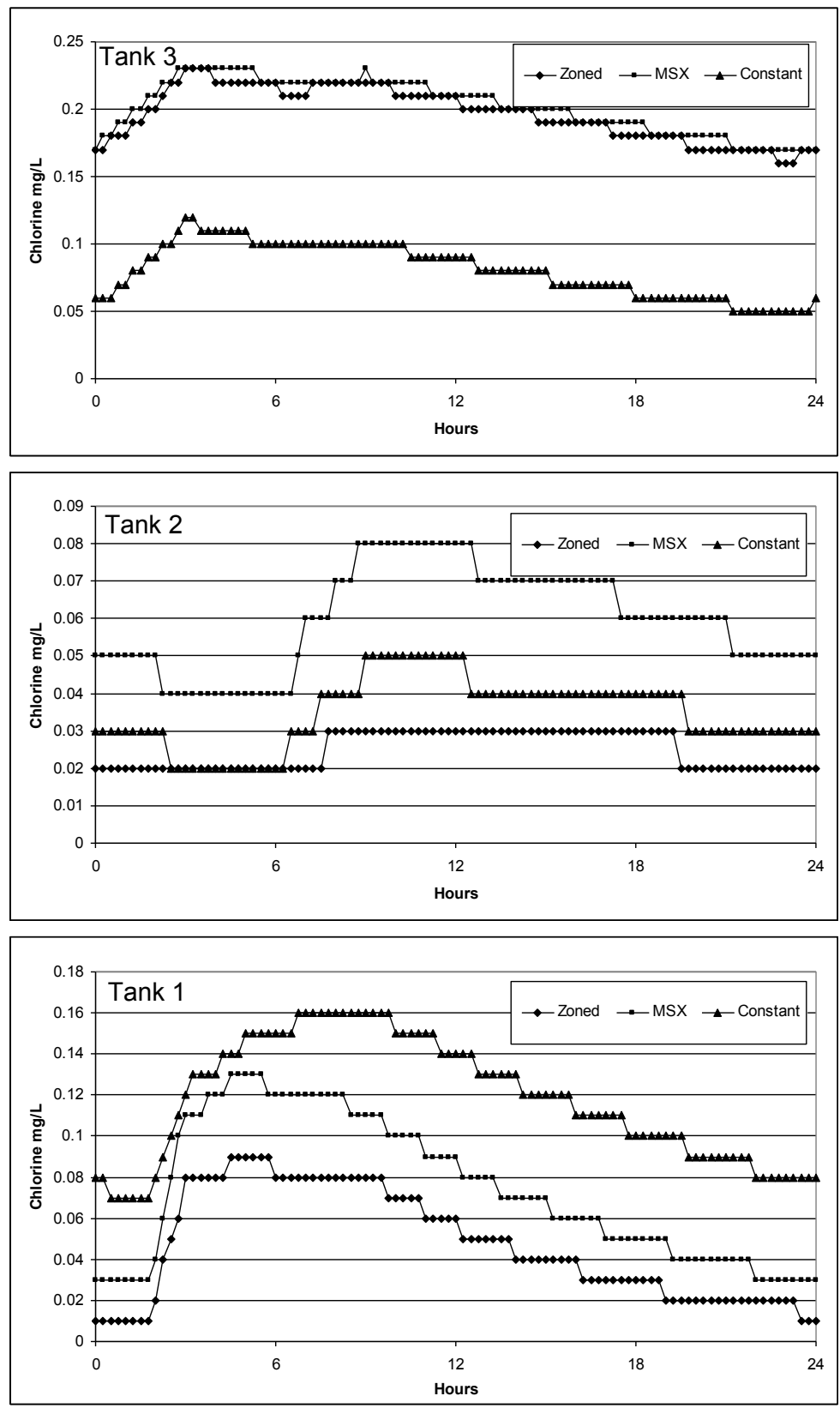

Figure 21.4 Modeled chlorine residual at tanks using alternative models for bulk decay. 

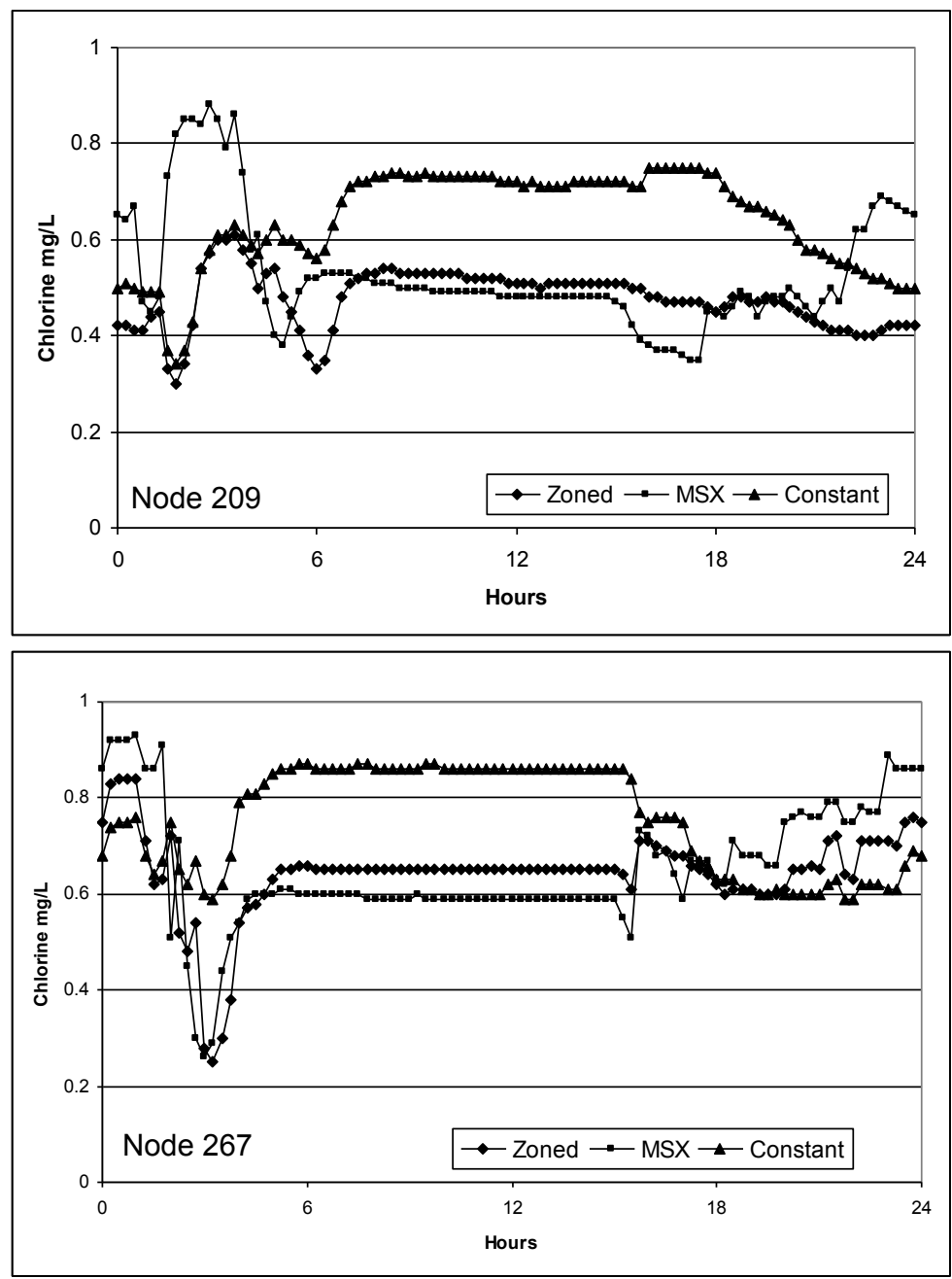

Figure 21.5 Modeled chlorine residual at selected nodes using alternative models for bulk decay.

Table 21.1 Range of modeled chorine residual for selected locations.

\begin{tabular}{lccc}
\hline \multicolumn{1}{c}{ Location } & Global Model & Zoned Model & MSX Model \\
\hline Tank 1 & $0.07-0.16$ & $0.01-0.09$ & $0.03-0.13$ \\
Tank 2 & $0.02-0.05$ & $0.02-0.03$ & $0.04-0.08$ \\
Tank 3 & $0.06-0.12$ & $0.17-0.24$ & $0.17-0.24$ \\
Node 267 & $0.59-0.87$ & $0.25-0.71$ & $0.26-0.73$ \\
Node 209 & $0.34-0.75$ & $0.30-0.61$ & $0.35-0.88$ \\
\hline
\end{tabular}


As illustrated, the variations in results among the three methods are quite different for the three locations.

For tank 1, which is located in the central part of the system and receives differing percentages of water from the two sources over the course of the day, the three methods give significantly different results. The global method results in the highest range of chlorine residuals, the zoned method results in the lowest range, and the MSX model results in an intermediate range.

For tank 2, which is located in the southern part of the system and also receives varying percentages of water from the two sources, a similar disparity in results occurs. However, in this case, the MSX model results in the highest range, the zoned in the lowest range and the global method results in intermediate values.

Tank 3 is located in the northern portion of the system and receives almost all of its water from the river source. As a result, the MSX model and the zoned model give almost identical results and the global model predicts a much lower range of chlorine residuals.

For the nodes, the chlorine residuals were higher than at the tanks because much of the water reaches the nodes without going through the tanks. At both nodes, the zoned model and the MSX model gave relatively similar results while the global (constant) model results varied significantly.

\subsection{Future Work}

The method described in this chapter will be applied to a distribution system at an army base located in northeastern United States. The distribution system at the army base is served from two sources, a surface water source and a groundwater source, that have very significant differences in their water quality characteristics. Furthermore, there are significant daily and seasonal variations in how the water system is operated. As a result, the fraction of water from each source reaching any node in the system may vary from hour to hour, on a daily basis, or from season to season.

In order to support the modeling on the army base, chlorine bottle tests have been performed and additional tests will be performed in the future in order to determine the bulk chlorine decay associated with each source. Additional tests will be performed to determine the bulk chlorine decay rate associated with water blended from the two sources. Limited test results for blended water for a water system in California suggest that the proposed linear combination of bulk decay rates based on the fraction of water from multiple sources provides a reasonable representation (Vasconcelos et al., 1996). However, further testing is needed to confirm or to refine the linear assumption. 
Comparison of modeled and measured field chlorine values are planned to help calibrate and validate the model. This will include estimation of chlorine wall decay coefficients.

\subsection{Conclusions}

The work described in this chapter is a preliminary proof of concept of the use of EPANET MSX as a means of more accurately modeling chlorine residual in distribution systems with multiple sources. The results indicate that there can be significant differences in modeled chlorine residuals in the distribution system when different methods are used for assigning bulk chlorine decay coefficients. The described approach allows the modeler to incorporate the variability in the bulk decay coefficient caused by blending into the standard chlorine modeling equations. Further work is needed to study the modeling improvements resulting from such adaptations.

\section{References}

Rossman, L.A., Clark, R.M. and Grayman, W.M. 1994. Modeling Chlorine Residuals in Drinking Water Distribution Systems. Journal of Environmental Engineering, 120(4): 803-20.

Shang, F., Uber, J.G. and Rossman, L.A. 2008. EPANET Multi-Species Extension User's Manual. USEPA Office of Research and Development - National Homeland Security Research Center. EPA/600/S-07/021. Revised October 2008.

Vasconcelos, J.J., Boulos, P.F., Grayman, W.M., Kiene, L., Wable, O., Biswas, P., Bhari, A., Rossman, L.A., Clark, R.M. and Goodrich, J.A. 1996. Characterization and Modeling of Chlorine Decay in Distribution Systems. American Water Works Research Foundation. Denver, Colorado. 